\title{
Sigmoid Colon Varices due to Massive Thrombosis of a Noncirrhotic Extrahepatic Portosystemic Shunt
}

\author{
Manabu Hayashi ${ }^{1}$, Tatsuro Sugaya ${ }^{1}$, Masashi Fujita ${ }^{1}$, Jun Nakamura ${ }^{1,2}$, Hiromichi Imaizumi ${ }^{1,3}$, \\ Kazumichi Abe ${ }^{1}$, Atsushi Takahashi ${ }^{1}$, Tadayuki Takagi ${ }^{1}$, Takuto Hikichi ${ }^{2}$ and Hiromasa Ohira ${ }^{1}$
}

\begin{abstract}
:
A 33-year-old man presented with hepatic encephalopathy and was diagnosed to have a noncirrhotic extrahepatic portosystemic shunt (NCPSS). He presented with abdominal pain 16 months after the NCPSS diagnosis. Computed tomography revealed thrombosis between the intrahepatic portal vein and the left internal iliac vein, including the NCPSS, and varices of the sigmoid colon. Thrombosis was treated with danaparoid sodium and antithrombin III followed by edoxaban. After treatment, the thrombosis disappeared from the intrahepatic portal vein, but it remained in the NCPSS. The sigmoid colon varices were followed up without any treatment. Follow-up is needed in NCPSS patients in order to make an early detection of complications.
\end{abstract}

Key words: congenital portosystemic shunt, portal vein thrombosis, ectopic varices, danaparoid

(Intern Med 59: 2705-2710, 2020)

(DOI: 10.2169/internalmedicine.4925-20)

\section{Introduction}

A congenital portosystemic shunt (CPSS) is a rare congenital venous anomaly between the portal vein and the systemic venous circulation that can be diagnosed at any age (1). A CPSS is a noncirrhotic extrahepatic portosystemic shunt (NCPSS). A CPSS without a portal vein flow in the liver is classified as type I, and a CPSS preserving some hepatic portal flow is classified as type II (2). Liver transplantation may be needed for CPSS type I patients with refractory symptoms (3). Symptomatic CPSS type II patients are treated with embolization by either operative ligation or balloon-occluded retrograde transvenous obliteration (BRTO). PSS closure may decrease the risk of complications (4). The clinical course in CPSS type II patients varies widely and may include jaundice, hepatic encephalopathy (HE), and bleeding (4).

Malignancy unrelated to portal vein thrombosis (PVT) commonly occurs in patients with liver cirrhosis (5). However, PVT is not common in NCPSS patients, and the clinical course of these patients is unknown. We herein report the case of a patient with massive thrombosis of an NCPSS.
He developed secondary varices in the sigmoid colon, but he did not show any variceal bleeding during the follow-up period.

\section{Case Report}

A 33-year-old man was referred to a local hospital with a loss of consciousness. He was confused and developed seizures after consuming alcohol. Blood tests showed hyperammonemia, and symptoms were improved after intravenous fluid therapy. His height was $186 \mathrm{~cm}$, and his body weight was $93 \mathrm{~kg}$. He did not have a history of excessive alcohol intake. He sometimes showed bloody stool due to hemorrhoids. He did not have any other specific medical histories. Computed tomography (CT) revealed a PSS between the splenic vein and the left iliac vein with an almost normal liver (Fig. 1A, B). The maximum diameter of the PSS was $46 \mathrm{~mm}$. The main portal vein was narrow compared with the PSS. There were no findings of splenomegaly. Magnetic resonance imaging showed hyperintensity in the bilateral globus pallidus on T1-weighted imaging (Fig. 1C). The patient was referred to our hospital and underwent liver biopsy. The liver histology was almost normal, and there was

${ }^{1}$ Department of Gastroenterology, Fukushima Medical University, Japan, ${ }^{2}$ Department of Endoscopy, Fukushima Medical University Hospital, Japan and ${ }^{3}$ Department of Internal Medicine, Iwase General Hospital, Japan

Received: March 24, 2020; Accepted: May 25, 2020; Advance Publication by J-STAGE: July 14, 2020

Correspondence to Dr. Manabu Hayashi, m884884@fmu.ac.jp 

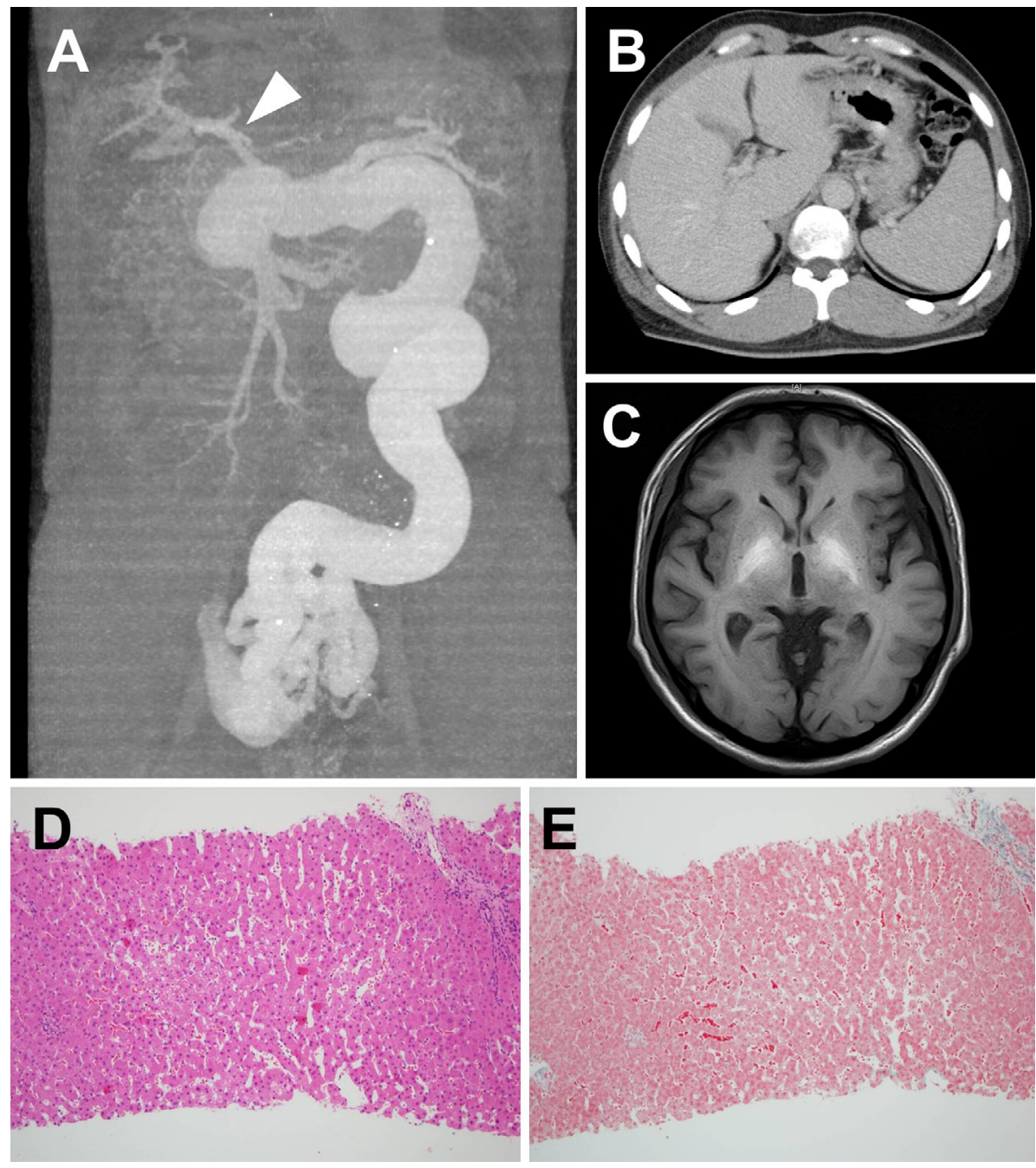

Figure 1. A large portosystemic shunt between the splenic vein and the left iliac vein on CT angiography of the portal vein (A) and almost normal liver findings (B). The main portal vein was narrow (A, white arrowhead). Hyperintensity at the bilateral globus pallidus on T1-weighted MRI (C). No evidence of cirrhosis or parenchymal injury in the liver (D: Hematoxylin and Eosin staining, $\times 100$. E: Elastica-Masson staining, $\times$ 100).

no evidence of either cirrhosis or parenchymal injury (Fig. 1D, E). The laboratory findings did not show any elevated liver enzymes (see 1st admission in Table) or signs of chronic liver disease, such as positivity for hepatitis $\mathrm{C}$ virus antibody, hepatitis B surface antigen, or antinuclear antibody. The lupus anticoagulant and anticardiolipin antibody findings were also negative. Protein $\mathrm{C}$ activation was low compared with the the normal range. He was diagnosed with HE due to CPSS type II. We considered embolization of the PSS by B-RTO, but the femoral vein approach to the shunt was deemed to be difficult. Furthermore, we did not possess an appropriate balloon-occlusion catheter to embolize such a large PSS. Operative ligation was not selected by the patient. The patient was therefore treated conservatively with lactulose.

Sixteen months after the CPSS diagnosis, the patient developed abdominal pain and bloody stools. He also had a fever $\left(37.6{ }^{\circ} \mathrm{C}\right.$ ) and systemic edema (his body weight was $101 \mathrm{~kg}$ ). CT revealed thrombosis between the intrahepatic portal vein and the left ileac vein, including the PSS (Fig. 2A-C). Varices had developed in the sigmoid colon (Fig. 3A). Intestinal edema (Fig. 2B, D) and pleural effusion were observed. Colonoscopy showed hemorrhoids, which were the cause of the bloody stool. There were small sigmoid colon varices (Fig. 3D). The laboratory findings showed elevated coagulation and inflammation markers (see 2nd admission in Table). The PVT was treated with intravenous danaparoid sodium and antithrombin III (ATIII) followed by edoxaban. Antibacterial treatment using carbapenem was administered because pylephlebitis was suspected. However, two sets of blood cultures were negative. The abdominal pain, fever, and bloody stool improved. He was discharged 25 days after admission and was followed with edoxaban treatment. One month after discharge, his protein $\mathrm{C}$ activation levels improved to within the normal range (65\%). Thrombosis in the intrahepatic portal vein disappeared 3 months after treatment (Fig. 2E). However, there was no reduction in the thrombosis in the PSS. The size of 
Table. Laboratory Findings.

\begin{tabular}{|c|c|c|}
\hline & 1st admission & 2nd admission \\
\hline \multicolumn{3}{|l|}{ Complete blood count } \\
\hline White blood cell count $\left(/ \mathrm{mm}^{3}\right)$ & 6,500 & 25,400 \\
\hline Red blood cell count $\left(\times 10^{4} / \mathrm{mm}^{3}\right)$ & 558 & 377 \\
\hline Hemoglobin level (g/dL) & 13.7 & 7.9 \\
\hline Platelet count $\left(\times 10^{4} / \mathrm{mm}^{3}\right)$ & 14.0 & 8.4 \\
\hline \multicolumn{3}{|l|}{ Coagulation status } \\
\hline Prothrombin activity (\%) & 61.0 & 71.9 \\
\hline Activated partial thromboplastin time (sec) & 37.0 & 36.9 \\
\hline D-dimer $(\mu \mathrm{g} / \mathrm{mL})$ & & 71.1 \\
\hline Fibrin degradation products $(\mu \mathrm{g} / \mathrm{mL})$ & & 113.9 \\
\hline Thrombin-antithrombin complexes (ng/mL) & & 6.0 \\
\hline Plasmin $\alpha 2$ plasmin inhibitor complex $(\mu \mathrm{g} / \mathrm{mL})$ & & 3.6 \\
\hline Antithrombin III (\%) & & 61 \\
\hline Protein $S$ activation (\%) (normal range: $67-164)$ & 70 & 68 \\
\hline Protein $\mathrm{C}$ activation (\%) (normal range: $64-140$ ) & 47 & 42 \\
\hline \multicolumn{3}{|l|}{ Biochemistry } \\
\hline Aspartate transaminase (U/L) & 32 & 23 \\
\hline Alanine aminotransferase $(\mathrm{U} / \mathrm{L})$ & 27 & 18 \\
\hline Lactate dehydrogenase (U/L) & 263 & 434 \\
\hline Alkaline phosphatase (U/L) & 278 & 177 \\
\hline$\gamma$-Glutamyl transpeptidase (U/L) & 22 & 20 \\
\hline Total protein $(\mathrm{g} / \mathrm{dL})$ & 5.0 & 4.2 \\
\hline Albumin $(\mathrm{g} / \mathrm{dL})$ & 2.4 & 1.2 \\
\hline Total bilirubin (mg/dL) & 0.7 & 0.8 \\
\hline Blood urea nitrogen $(\mathrm{mg} / \mathrm{dL})$ & 14 & 36 \\
\hline Creatinine $(\mathrm{mg} / \mathrm{dL})$ & 0.88 & 0.81 \\
\hline Ammonia $(\mu \mathrm{g} / \mathrm{mL})$ & 113 & 39 \\
\hline C-reactive protein $(\mathrm{mg} / \mathrm{dL})$ & 0.06 & 14.9 \\
\hline Hepatitis $\mathrm{C}$ antibody (S/CO) & 0.1 & \\
\hline Hepatitis B surface antigen (IU/mL) & 0.00 & \\
\hline Antinuclear antibody & $<80 x$ & \\
\hline
\end{tabular}

the sigmoid varices gradually increased (Fig. 3), but there were no high-risk findings of variceal bleeding on colonoscopy, and he did not show symptoms of variceal bleeding. The sigmoid colon varices were followed but not treated. There was no recurrence of intrahepatic portal vein thrombosis, and the thrombosis in the PSS did not show recanalization at 10 months after treatment. The patient's serum manganese concentration was $0.2 \mu \mathrm{g} / \mathrm{dL}$ (normal range: $<0.8$ $\mu \mathrm{g} / \mathrm{dL}$ ), and he did not show either HE or variceal bleeding 12 months after undergoing treatment for thrombosis.

\section{Discussion}

An NCPSS is a relatively rare condition (2). Although a PSS is commonly observed in patients with liver cirrhosis, the liver histology in this case did not show any evidence of cirrhosis. Idiopathic portal hypertension (IPH) may partially include an NCPSS (6). IPH is clinically characterized by features of portal hypertension of an unknown cause and often shows the presence of a PSS (7). In this case, the patient showed a normal liver histology. There were no findings of either periportal fibrosis or aberrant vessels in the portal tract, which suggested the presence of IPH. The main portal vein in IPH patients is often dilated, but the main portal vein in our patient was narrow (7). Furthermore, he did not have any specific medical history or systemic disorder that may have caused IPH or intrahepatic portal vein atrophy. The narrowness of his portal vein was thought to be a congenital abnormality. The hyperintensity in his bilateral globus pallidus on T1-weighted imaging by MR imaging might be associated with high manganese concentrations caused by the PSS (8). His serum manganese concentrations may have decreased after the development of thrombosis in the PSS, as the levels of $\mathrm{NH}_{3}$ and protein $\mathrm{C}$ both improved after the development of thrombosis in the PSS. Further, it has been reported that plasma manganese does not show a significant correlation with the signal intensity of the globus pallidus on T1-weighted MRI in cirrhotic patients (9).

In our case, the patient was diagnosed with an NCPSS based on the development of HE. Treatment for HE in patients with an NCPSS is important. The development of HE is related to an impaired quality of life (10). Falls and poor driving are also related to $\operatorname{HE}(11,12)$. Generally, we consider PSS closure in patients who develop refractory HE. A 


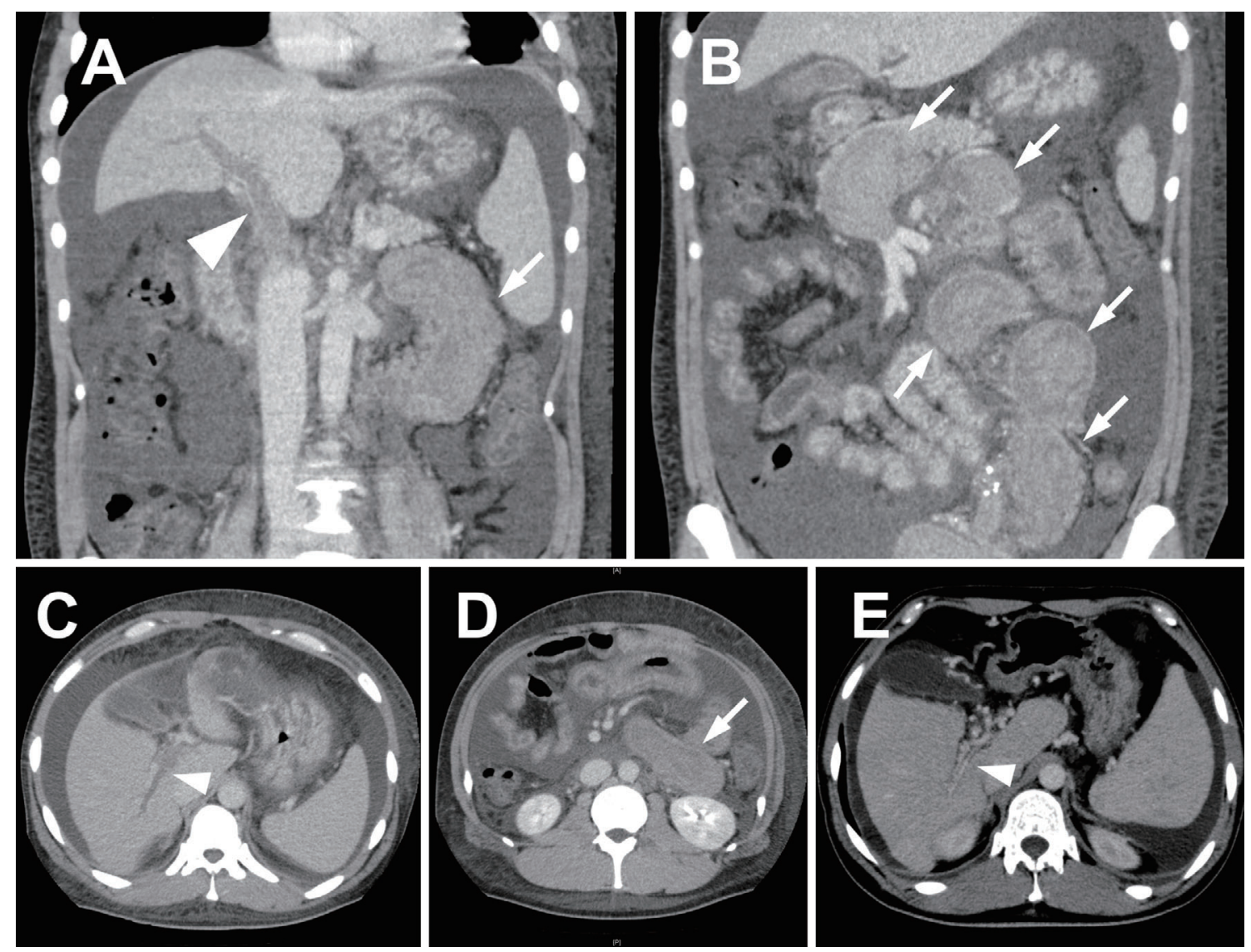

Figure 2. Thrombosis in the portosystemic shunt on coronal (A, B) and sagittal (C, D) CT. Thrombosis in the intrahepatic portal vein (A, C, white arrowheads) and in the portosystemic shunt (A-D, white arrows). Thrombosis in the intrahepatic portal vein disappeared 3 months after treatment (E, white arrowhead).

large PSS was evident in our patient, and a new collateral vein developed due to PSS thrombosis. These findings suggest that the blood flow of a large PSS may be inappropriate for narrow intrahepatic portal veins. The rapid deterioration of portal hypertension by PSS ligation might result in the development of ascites or a worsening of the liver function. On the other hand, there was a report of successful closure in adult CPSS patient (13). Operative ligation in pediatric CPSS patients has been reported to be safe and effective (4). If the HE had proved to be uncontrollable by conservative treatment, then we might have considered the performance of an elective PSS closure.

Virchow's triad, i.e., hypercoagulability, endothelial injury, and a reduced blood flow, is an important factor in the development of venous thrombosis (14). In this case, the low activity of protein $\mathrm{C}$ and the large PSS may have been associated with the development of massive thrombosis. A low protein $\mathrm{C}$ level is associated with the development of venous thrombosis, and a high prevalence of protein $\mathrm{C}$ deficiency has been reported in patients with splenic vein thrombosis (15). A large PSS may slow the blood flow in the portal vein. A slow blood flow is a risk factor for PVT (16) and it may lead to an impaired thrombin breakdown (17). Acute symptomatic PVT requires urgent management, and treatment failure can result in mesenteric ischemia $(18,19)$. Sev- eral anticoagulant agents are used to treat PVT in cirrhotic patients. A recent report demonstrated the utility of intravenous danaparoid and ATIII followed by edoxaban for PVT in cirrhotic patients $(20,21)$. Although our patient was not cirrhotic, the administration of danaparoid and ATIII followed by edoxaban was effective for treating intrahepatic PVT and preventing PSS thrombosis progression.

Diffuse varices in the sigmoid colon developed due to massive thrombosis in the PSS. The endoscopic findings can be used to stratify the risk of bleeding in gastrointestinal varices. The red color sign is useful for predicting the first onset of bleeding from esophageal and rectal varices $(22,23)$. In our patient, the sigmoid colon varices did not show a red color sign, and the patient did not develop variceal bleeding. However, alterations in hemodynamics due to thrombosis may result in a deterioration of the varices. Evaluation by CT and endoscopy is needed in the follow-up period. If varices in the sigmoid colon showed signs of high-risk bleeding (either enlargement or the red color sign), then we consider prophylactic endoscopic intervention, such as endoscopic injection sclerosis (EIS) or endoscopic variceal ligation (EVL). There have been reports that both EIS and EVL are effective for the treatment of varices of the rectum (24). Although it is unknown which procedure is preferable, we prefer to perform EIS using 


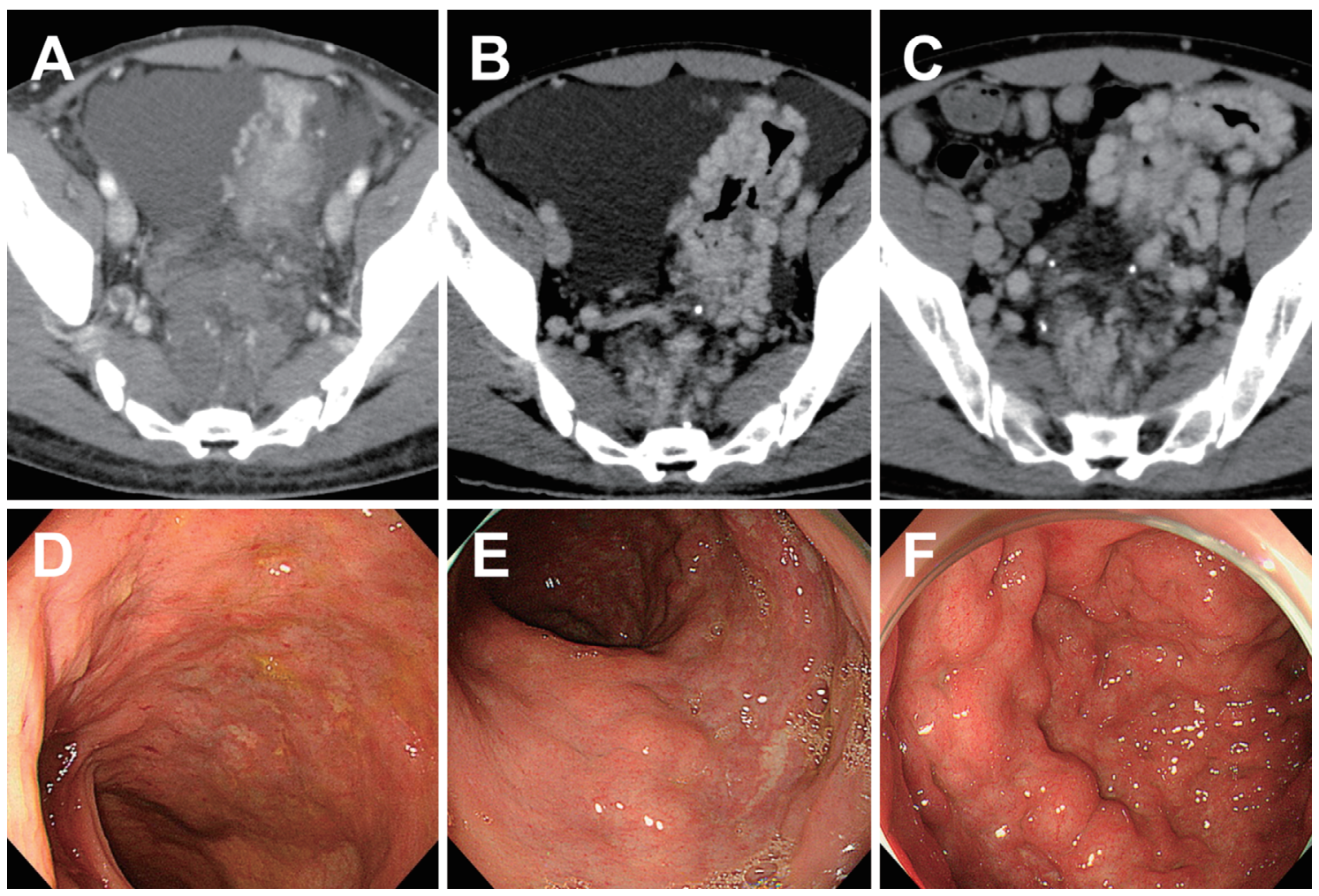

Figure 3. Findings of sigmoid colon varices on CT (A-C) and colonoscopy (D-F) at the time of thrombosis development (A, D), 3 months after treatment (B, E), and 10 months after treatment (C, F).

either the ethanolamine oleate method or n-butyl-2cyanoacrylate method, as there might be a risk of wideranging mucosal defects on the varices in the event of insufficient variceal ligation.

In summary, we herein described a case of massive NCPSS thrombosis, which was thought to be a congenital anomaly. Our case suggests that follow-up is needed in NCPSS patients for the early detection of complications, such as the development of PVT or ectopic varices. Furthermore, the hemodynamics can be complicated and vary widely among NCPSS patients. The treatment for an NCPSS, especially invasive interventions, should be carefully considered on a case-by-case basis for each patient.

The authors state that they have no Conflict of Interest (COI).

\section{References}

1. McLin VA, Franchi Abella S, Debray D, et al. Congenital portosystemic shunts: current diagnosis and management. J Pediatr Gastroenterol Nutr 68: 615-22, 2019.

2. Morgan G, Superina R. Congenital absence of the portal vein: two cases and a proposed classification system for portasystemic vascular anomalies. J Pediatr Surg 29: 1239-1241, 1994.

3. Ohnishi Y, Ueda M, Doi H, et al. Successful liver transplantation for congenital absence of the portal vein complicated by intrapulmonary shunt and brain abscess. J Pediatr Surg 40: e1-e3, 2005.

4. Lautz TB, Tantemsapya N, Rowell E, Superina RA. Management and classification of type II congenital portosystemic shunts. J Pe- diatr Surg 46: 308-314, 2011.

5. Intagliata NM, Caldwell SH, Tripodi A. Diagnosis, development, and treatment of portal vein thrombosis in patients with and without cirrhosis. Gastroenterology 156: 1582-1599 e1, 2019.

6. Khanna R, Sarin SK. Non-cirrhotic portal hypertension - diagnosis and management. J Hepatol 60: 421-441, 2014.

7. Hernandez-Gea V, Baiges A, Turon F, Garcia-Pagan JC. Idiopathic portal hypertension. Hepatology 68: 2413-2423, 2018.

8. Rovira A, Alonso J, Cordoba J. MR imaging findings in hepatic encephalopathy. AJNR Am J Neuroradiol 29: 1612-1621, 2008.

9. Choi Y, Park JK, Park NH, et al. Whole blood and red blood cell manganese reflected signal intensities of T1-weighted magnetic resonance images better than plasma manganese in liver cirrhotics. J Occup Health 47: 68-73, 2005.

10. Prasad S, Dhiman RK, Duseja A, Chawla YK, Sharma A, Agarwal R. Lactulose improves cognitive functions and health-related quality of life in patients with cirrhosis who have minimal hepatic encephalopathy. Hepatology 45: 549-559, 2007.

11. Soriano G, Roman E, Cordoba J, et al. Cognitive dysfunction in cirrhosis is associated with falls: a prospective study. Hepatology 55: 1922-1930, 2012.

12. Kircheis G, Knoche A, Hilger N, et al. Hepatic encephalopathy and fitness to drive. Gastroenterology 137: 1706-1715 e1-e9, 2009.

13. Elnekave E, Belenky E, Van der Veer L. Noncirrhotic extrahepatic portosystemic shunt causing adult-onset encephalopathy treated with endovascular closure. Case Rep Radiol 2015: 852853, 2015.

14. Valla DC, Rautou PE. The coagulation system in patients with end-stage liver disease. Liver Int 35 (Suppl 1): 139-144, 2015.

15. Sutkowska E, McBane RD, Tafur AJ, et al. Thrombophilia differences in splanchnic vein thrombosis and lower extremity deep venous thrombosis in North America. J Gastroenterol 48: 1111-1118, 2013. 
16. Tsochatzis EA, Senzolo M, Germani G, Gatt A, Burroughs AK. Systematic review: portal vein thrombosis in cirrhosis. Aliment Pharmacol Ther 31: 366-374, 2010.

17. Zocco MA, Di Stasio E, De Cristofaro R, et al. Thrombotic risk factors in patients with liver cirrhosis: correlation with MELD scoring system and portal vein thrombosis development. J Hepatol 51: 682-689, 2009.

18. Berzigotti A, Garcia-Criado A, Darnell A, Garcia-Pagan JC. Imaging in clinical decision-making for portal vein thrombosis. Nat Rev Gastroenterol Hepatol 11: 308-316, 2014.

19. Hunninghake J, Murray BP, Ferraro D, Gancayco J. Acute intestinal ischaemia from a portal vein thrombosis in a young female smoker on an oral contraceptive. BMJ Case Rep 2018: 2018.

20. Nagaoki Y, Aikata H, Daijyo K, et al. Efficacy and safety of edoxaban for treatment of portal vein thrombosis following danaparoid sodium in patients with liver cirrhosis. Hepatol Res 48: 51-58, 2018.

21. Kojima S, Watanabe N, Koizumi J, et al. Current status of portal vein thrombosis in Japan: results of a questionnaire survey by the
Japan Society for Portal Hypertension. Hepatol Res 48: 244-254, 2018.

22. Shudo R, Yazaki Y, Sakurai S, Uenishi H, Yamada H, Sugawara K. Clinical study comparing bleeding and nonbleeding rectal varices. Endoscopy 34: 189-194, 2002.

23. Murachima N, Ikeda K, Kobayashi M, et al. Incidence of the appearance of the red color sign on esophageal varices and its predictive factors: long-term observations of 359 patients with cirrhosis. J Gastroenterol 36: 368-374, 2001.

24. Sato T, Yamazaki K, Toyota J, Karino Y, Ohmura T, Suga T. The value of the endoscopic therapies in the treatment of rectal varices: a retrospective comparison between injection sclerotherapy and band ligation. Hepatol Res 34: 250-255, 2006.

The Internal Medicine is an Open Access journal distributed under the Creative Commons Attribution-NonCommercial-NoDerivatives 4.0 International License. To view the details of this license, please visit (https://creativecommons.org/licenses/ by-nc-nd/4.0/).

(C) 2020 The Japanese Society of Internal Medicine

Intern Med 59: 2705-2710, 2020 